\title{
Mammalian sirtuins-emerging roles in physiology, aging, and calorie restriction
}

\author{
Marcia C. Haigis and Leonard P. Guarente ${ }^{1}$ \\ Department of Biology, Massachusetts Institute of Technology, Cambridge, Massacusetts 02139, USA
}

Sir2 is an NAD-dependent deacetylase that connects metabolism with longevity in yeast, worms and flies. Mammals contain seven homologs of yeast Sir2, SIRT1-7. Here, we review recent findings demonstrating the role of these mammalian sirtuins as regulators of physiology, calorie restriction, and aging. The current findings sharpen our understanding of sirtuins as potential pharmacological targets to treat the major diseases of aging.

Silent information regulator (Sir) proteins regulate lifespan in multiple model organisms. In yeast, an extra copy of the SIR2 gene extends replicative lifespan by $50 \%$, while deleting Sir2 shortens lifespan (Kaeberlein et al. 1999). Sir2 silences chromatin, enables DNA repair, and is involved in chromosome fidelity during meiosis (Blander and Guarente 2004). Sir2 promotes longevity by suppressing the formation of toxic extrachromosomal rDNA circles (ERCs) in yeast (Sinclair and Guarente 1997). The Caenorhabditis elegans ortholog sir-2.1 also extends worm lifespan (Tissenbaum and Guarente 2001), but by a distinct mechanism. Sir-2.1 requires the worm forkhead protein DAF-16 for lifespan extension (Tissenbaum and Guarente 2001). While earlier models suggested sir-2.1 might function by down-regulating insulin signaling, more recent findings show that sir-2.1 binds to DAF-16, activating it directly (Berdichevsky et al. 2006). Moreover, sir-2.1 does not respond to changes in insulin signaling, but, rather, is activated by stress treatments, such as heat shock and oxidative damage (Berdichevsky et al. 2006). Likewise, an increase in the dosage of Drosophila Sir2 extends lifespan (Rogina and Helfand 2004).

Although a link between Sir2 and longevity was clear, its enzymatic activity remained elusive for years. An early clue came from the observation that CobB, an Escherichia coli homolog of Sir2, could catalyze the phosphoribosyltransferase reaction in cobalamin biosynthesis (Tsang and Escalante-Semerena 1998). Thus, it was predicted and demonstrated that Sir2 possessed NAD-dependent ADP-ribosyltransferase activity (Frye

[Keywords: Sir2; aging; glutamate dehydrogenase; SIRT1-7; mitochondria; insulin; calorie restriction]

${ }^{1}$ Corresponding author.

E-MAIL leng@mit.edu; FAX (617) 253-8699.

Article is online at http://www.genesdev.org/cgi/doi/10.1101/gad.1467506.
1999; Tanny et al. 1999). Subsequent reports revealed that yeast and mammalian sirtuins catalyze a novel and robust reaction, NAD-dependent histone deacetylation, unavoidably linking Sir2 activity with metabolism (Imai et al. 2000; Landry et al. 2000; Smith et al. 2000). Mechanistically, ADP-ribosylation and deacetylation reactions by sirtuins are similar because they cleave NAD in each reaction cycle (Fig. 1) (Grubisha et al. 2005). During each cycle, deacetylation generates the novel metabolites, 2' and $3^{\prime}$-O-acetyl-ADP-ribose (Tanner et al. 2000; Sauve et al. 2001; Tanny and Moazed 2001), which may be important regulators of physiology (Grubisha et al. 2006). Today, more than a dozen nonhistone deacetylation substrates are known, several of which are described below.

Calorie restriction (CR) is a dietary regimen that extends the lifespan of every organism tested to date. Specifically, CR extends the lifespan of yeast (Lin et al. 2002), spiders (Austad 1989), flies (Loeb and Northrop 1917), fish (Comfort 1963), and rodents (McCay et al. 1935; Austad 1989). Sir2 is required for lifespan extension by CR in yeast, worms, and flies (Lin et al. 2000; Rogina and Helfand 2004; Wang and Tissenbaum 2006). In yeast, CR $(0.5 \%$ glucose), was previously shown to increase mitochondrial function and to up-regulate SIR2 activity (Lin et al. 2002, 2004). However, in this case, the mitochondrial activation is SIR2-independent, suggesting that it lies upstream of SIR2. A more severe CR regimen $(0.05 \%$ glucose $)$ extends yeast replicative lifespan by a different mechanism that is apparently independent of both SIR2 and mitochondrial respiration (Kaeberlein et al. 2004, 2005). Finally, SIR2 has no effect on yeast survival under starvation conditions, and appears to actually reduce survival of certain exceptionally long-lived mutant strains (Fabrizio et al. 2005).

Do sirtuins regulate human longevity? Mammals have seven Sir2 homologs (sirtuins, SIRT1-7). These proteins have a highly conserved NAD-dependent sirtuin core domain, first identified in the founding yeast SIR2 protein, making them good candidates as lifespan regulators (Frye 2000). As highlighted in this review, mammalian sirtuins have diverse cellular locations, target multiple substrates, and affect a broad range of cellular functions (Table 1). In this review, we emphasize an emerging theme in the field of aging - the regulation of oxidative stress, DNA damage, and metabolism by mammalian sirtuins. 


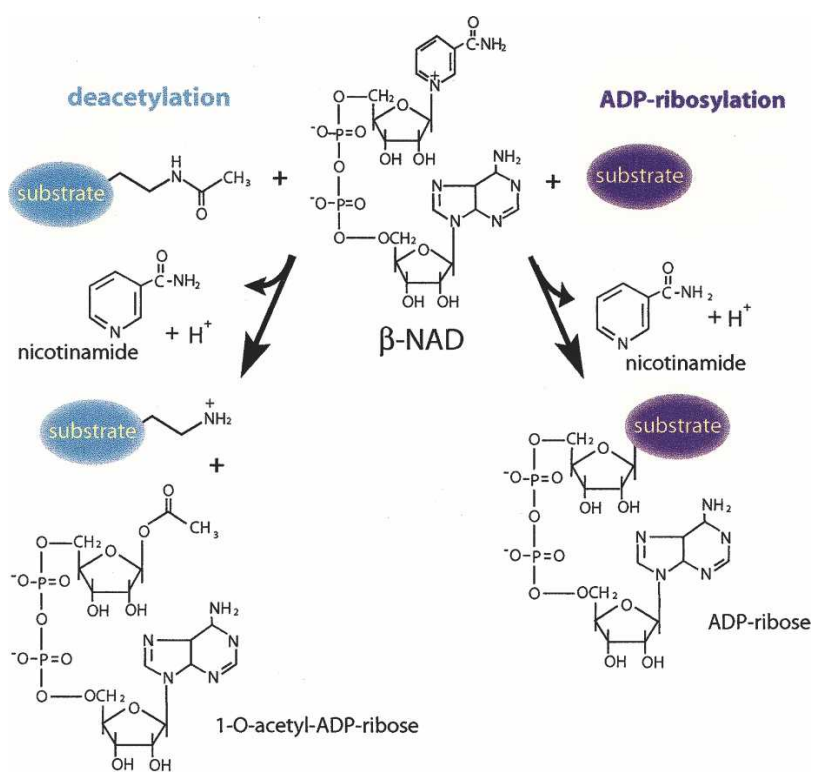

Figure 1. Sirtuin deacetylation and ADP-ribosylation reactions. Both deacetylation and ADP-ribosylation occur via cleavage of NAD to release nicotinamide.

\section{Nuclear sirtuins}

Three mammalian sirtuins (SIRT1, SIRT6, and SIRT7) are localized to the nucleus. SIRT1 is most extensively studied, has more than a dozen known substrates, and is a guardian against cellular oxidative stress and DNA damage. Moreover, SIRT1 plays a prominent role in metabolic tissues, such as pancreas, fat, and liver. SIRT6 and SIRT7 may also be important regulators of DNA damage and metabolism, respectively.

\section{SIRT1 substrates in mammals}

While a role for mammalian sirtuins in lifespan regulation has not been directly determined, evidence suggests that the Sir2 ortholog, SIRT1, may regulate many physiological processes known to be affected during aging and which are altered by CR (Fig. 2). SIRT1 deacetylates a large number of substrates, including p53, Ku70, NF-кB, and forkhead proteins to affect stress resistance in cells (Luo et al. 2001; Vaziri et al. 2001; Brunet et al. 2004; Cohen et al. 2004; Motta et al. 2004; Yeung et al. 2004), which may relate to the observed stress resistance con- ferred by CR. SIRT1 also regulates the activities of the nuclear receptor PPAR $\gamma$ and PGC- $\alpha$ (see below) to influence differentiation of muscle cells, adipogenesis, fat storage in white adipose tissue, and metabolism in the liver, suggesting a possible connection between this sirtuin and diets that promote leanness and longevity (Fulco et al. 2003; Picard et al. 2004; Rodgers et al. 2005). The observed induction of SIRT1 protein during CR is consistent with this idea (Cohen et al. 2004; Nisoli et al. 2005). These activities link SIRT1 to known physiological effects of CR, and suggest that this sirtuin may help mediate $\mathrm{CR}$ in mice.

\section{SIRT1 regulation of insulin and glucose homeostasis}

A critical component of the physiology of CR is increased insulin sensitivity and corresponding reductions in blood glucose and insulin levels (Barzilai et al. 1998; Dhahbi et al. 2001). Pancreatic $\beta$-cells help to maintain glucose homeostasis by secreting insulin in response to glucose. Metabolism of glucose in these cells by glycolysis generates pyruvate, which enters mitochondria where it can be converted to $\mathrm{CO}_{2}$ by the TCA cycle. $\mathrm{NADH}$ made by this metabolic process drives electron transport and ATP synthesis. The increased ATP/ADP ratio causes closure of $\mathrm{K}_{\mathrm{ATP}}$ channels and depolarizes the plasma membrane leading to an influx of $\mathrm{Ca}^{2+}$, which triggers fusion of secretory vesicles containing insulin to the cell membrane.

Two recent studies in mice have demonstrated that SIRT1 positively regulates glucose-stimulated insulin secretion in pancreatic $\beta$-cells (Moynihan et al. 2005; Bordone et al. 2006). $\beta$-Cell-specific SIRT1-overexpressing (BESTO) mice demonstrate increased insulin secretion in response to glucose (Moynihan et al. 2005). Conversely, $S_{R T 1^{-/-}}$mice or their isolated islets show blunted insulin secretion (Bordone et al. 2006). Both studies find that SIRT1 represses transcription of the mitochondrial uncoupling protein UCP-2 gene, which uncouples mitochondrial respiration from ATP production and reduces the proton gradient across the mitochondrial membrane. Thus, by blocking UCP-2 function, SIRT1 promotes more efficient energy generation. Indeed, BESTO islets demonstrate higher ATP levels (Moynihan et al. 2005), while islets from SIRT1 knockout (KO) mice do not elevate ATP production in response to glucose (Bordone et al. 2006). Interestingly, SIRT1-mediated repression of UCP-2 is alleviated by acute food deprivation

Table 1. Diversity of mammalian sirtuins

\begin{tabular}{|c|c|c|c|c|}
\hline Sirtuin & Activity & Location & Interactions & Biology \\
\hline SIRT1 & Deacetylase & Nucleus & $\mathrm{FOXO}, \mathrm{PGC}-1 \alpha$ & Cell survival/metabolism \\
\hline SIRT2 & Deacetylase & Cytosol & Tubulin, H4 & Cell cycle \\
\hline SIRT3 & Deacetylase & Mitochondria & AceCS2 & Thermogenesis/metabolism \\
\hline SIRT4 & ADP-ribosyl-transferase & Mitochondria & $\mathrm{GDH}$ & Insulin secretion/metabolism \\
\hline SIRT5 & Deacetylase & Mitochondria & ? & ? \\
\hline SIRT6 & ADP-ribosyl-transferase & Nucleus & DNA Pol $\beta$ & DNA repair \\
\hline SIRT7 & $?$ & Nucleolus & Pol I & rDNA transcription \\
\hline
\end{tabular}




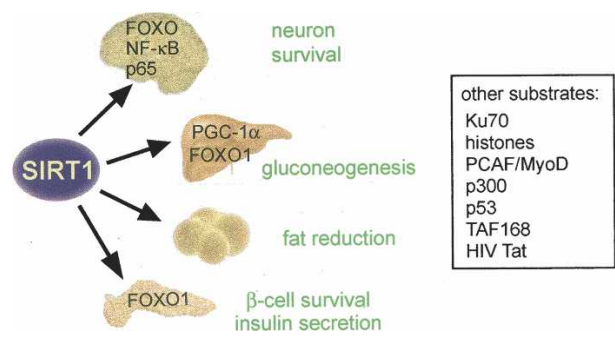

Figure 2. SIRT1 regulation of mammalian physiology. SIRT1 regulates neuron survival, gluconeogenesis, lipolysis, $\beta$-cell survival, and insulin secretion by interacting with a number of target proteins.

(Bordone et al. 2006), which may further dampen ATP synthesis and the insulin responsiveness of $\beta$-cells during starvation. While SIRT1 protein level is not affected by this condition, there is a decrease in the NAD/NADH ratio, which may reduce SIRT1 activity in pancreas (Bordone et al. 2006). The presence of UCP-2 in fasted animals may also ease the transition to metabolic activity after the next feeding and prevent hyperpolarization of the mitochondrial membrane and the corresponding production of reactive oxygen species. While these studies demonstrate that SIRT1 activity may be down-regulated in $\beta$-cells during fasting, it is not known whether SIRT1 regulates insulin secretion during $\mathrm{CR}$, or plays any role in pathologies demonstrating impaired insulin secretion.

Another study raises the possibility that SIRT1 promotes the survival of pancreatic $\beta$-cells during oxidative stress (Kitamura et al. 2005). In stressed $\beta$-cells, the forkhead protein FOXO1 moves into the nucleus and activates the $\beta$-cell transcription factors, NeuroD and MafA, and provides stress resistance. As described above, SIRT1 binds to and regulates forkhead transcription factors both negatively and positively (Brunet et al. 2004; Motta et al. 2004). Kitamura et al. (2005) show that nuclear FOXO1 associates with SIRT1 in PML (promyelocytic leukemia) bodies in stressed cells. They suggest that SIRT1 deacetylates FOXO1 at that location to activate the protein and provide stress resistance (Accili and Arden 2004). This process bears similarity to sir-2.1 in $C$. elegans discussed above, which functions as a coactivator of the DAF-16 protein after it translocates to the nucleus in oxidatively or heat stressed worms (Berdichevsky et al. 2006).

Glucose homeostasis is maintained by the liver, in addition to pancreatic $\beta$-cells, in response to changing nutrient conditions. During fasting, hepatocytes induce gluconeogenesis to supply other tissues with glucose. Several new studies have revealed that this nutrient response is under tight control of SIRT1 activity, providing another link between SIRT1 and metabolism. In cultured hepatocytes, SIRT1 interacts with and deacetylates nuclear FOXO1, promoting FOXO1-dependent transcription of hepatic gluconeogenic genes upon stress (Frescas et al. 2005). In the liver, the transcriptional coactivator PGC-1 $\alpha$ also drives expression of the gluconeogenic pathway. SIRT1 deacetylates and activates PGC-1 $\alpha$ to coordinate the increase in expression of gluconeogenic genes with the repression of glycolytic genes during fasting (Rodgers et al. 2005). However, in a neuronal cell line, overexpression of SIRT1 decreases the activity of PGC1- $\alpha$ and mitochondrial function (Nemoto et al. 2005), suggesting that the relationship between SIRT1 and PGC- $1 \alpha$ may be complex.

\section{SIRT1 and neuron function}

SIRT1 has also been linked to the survival of neurons. It is interesting to note that CR protects against neurodegenerative pathology in mouse models for Alzheimer's (Zhu et al. 1999; Patel et al. 2005) and Parkinson's (Duan and Mattson 1999). SIRT1 can promote survival in cultured neuronal cells as an antiapoptotic factor, perhaps through down-regulating the proapoptotic factors, p53 (Luo et al. 2001; Vaziri et al. 2001) and FOXO (Brunet et al. 2004; Motta et al. 2004). Even more interestingly, SIRT1 may be involved in the axonal protection observed in the Wallerian strain of mice (Araki et al. 2004), which have a translocation that increases levels of the NAD biosynthetic enzyme nicotinamide mononucleotide adenylyl-transferase 1 and renders peripheral axons more stable after a neuronal insult. Indeed, NAD itself provides protection to axons in cultured dorsal root ganglia. One study shows that the effects of NAD and the Wallerian strain are dependent on SIRT1, leading to the conclusion that this sirtuin is neuroprotective (Araki et al. 2004). However, another study did not observe a difference in the response of dorsal root ganglion from SIRT1 KO mice (Wang et al. 2005). It is interesting to note that the time course of axonal degradation in the two studies is different, suggesting that there may be two different neuroprotective processes induced by NADone dependent on SIRT1 and one not. Future studies should resolve these apparent experimental discrepancies.

SIR2 orthologs can also protect against neuronal dysfunction due to polyglutamine toxicity in C. elegans and mammalian cells (Parker et al. 2005). One study shows that neurotoxicity in worms is spared by the age- $1 \mathrm{mu}-$ tation, which reduces insulin-like signaling (Morley et al. 2002), or in a transgenic strain overexpressing sir-2.1 (Parker et al. 2005). Like the effects of the age-1 or sir-2.1 transgenes in extending lifespan, neuroprotection requires the forkhead protein DAF-16 (Parker et al. 2005). As might be expected, polyglutamine toxicity is exacerbated in daf-16 or sir-2.1 mutants. The putative SIR2activating polyphenol, resveratrol (Howitz et al. 2003), also protects against cell death in striatal neurons with the Huntingtons Disease allele htt (109Q) (Parker et al. 2005). Finally, $\beta$-amyloid-induced death of microglia is spared by overexpression of SIRT1 or resveratrol treatment (J. Chen et al. 2005). In toto, the above studies raise the possibility that activation of SIRT1 may be a novel strategy to protect against neurodegenerative diseases. We note, however, that the protective effect of SIRT1 may not be universal in all cell types, since SIRT1 ${ }^{-/}$ mouse embryonic fibroblasts (MEFs) actually survive 
better in culture and bypass senescence /Chua et al. 2005).

\section{SIRT1 and CR}

The above studies suggest that important physiological processes triggered by CR in mammals are regulated by SIRT1, making it vital to know whether mammalian sirtuins also regulate changes during CR. Recent experiments have directly related SIRT1 function to CR in mice. CR induces the endothelial nitric oxide synthase (eNOS), and results in an increase in mitochondrial biogenesis (Nisoli et al. 2005). Moreover, this mitochondrial induction by CR does not occur in eNOS deficient mice. Interestingly, the SIRT1 gene is activated by NO in vivo and in vitro (Nisoli et al. 2005), tracing a pathway in which CR induces NO production and activates mitochondrial biogenesis and SIRT1.

A more direct demonstration of the requirement of SIRT1 in CR involved placing SIRT1 KO mice on this diet (D. Chen et al. 2005). Although the KO mice show changes in blood glucose, triglycerides, and IGF-1 similar to wild-type controls, there is a large difference in one interesting output of CR. Wild-type mice show a fivefold to 10-fold increase in physical activity, which has been observed previously and may represent a foraging instinct induced by food insufficiency, but KO mice do not display any increase in activity. SIRT1 KO mice move as well or better than wild type when challenged by other means; i.e., rotarod or treadmill. This study indicates the first requirement for SIRT1 for at least one phenotype triggered by mammalian CR.

\section{SIRT6 regulates DNA repair}

SIRT6 is a nuclear protein widely expressed in mouse tissues (Liszt et al. 2005; Michishita et al. 2005). Original reports demonstrated that SIRT6 has a weak to absent in vitro deacetylate activity (North et al. 2003; Liszt et al. 2005). However, SIRT6 has also been shown to demonstrate a robust auto-ADP-ribosyltransferase activity (Liszt et al. 2005).

Recent work has provided insight into the diverse physiological functions of SIRT6 (Mostoslavsky et al. 2006). SIRT6 KO mice display premature aging symptoms, including loss of subcutaneous fat and decreased bone density, and die within 4 wk after birth. These phenotypes contrast with those of SIRT1 KO animals, which are postnatal lethal on an inbred strain (Cheng et al. 2003; McBurney et al. 2003). Outbred SIRT1 KO animals can survive into adulthood, but demonstrate a severe phenotype, including small size, delayed bone mineralization, defective skeletal closure, delayed eyelid opening, and sterility (Cheng et al. 2003; McBurney et al. 2003; Lemieux et al. 2005).

SIRT6 KO mice exhibit a deficiency in one specific form of DNA repair, the base excision repair (BER) (Mostoslavsky et al. 2006). MEFs lacking SIRT6 demonstrate impaired proliferation and enhanced sensitivity to DNAdamaging agents. SIRT6 KO MEFs demonstrate genomic instability in the form of chromosomal translocations, fragments, gaps, and detached centromeres. These defects can be rescued by overexpression of the DNA polymerase involved in BER, Polß. Furthermore, SIRT6 KO MEFs exhibit normal cell cycle checkpoints, end-joining, and double-strand break DNA repair. How SIRT6 regulates BER is still unknown. One might hypothesize that this sirtuin ADP-ribosylates a substrate protein involved in BER, which could be a component of the repair machinery or the chromatin at the site of DNA damage.

SIRT6 KO mice also display interesting metabolic phenotypes: low levels of circulating IGF-1 and hypoglycemia that becomes progressively more severe with age (Mostoslavsky et al. 2006). It will be interesting to know whether these metabolic changes are due to a direct role for SIRT6 in regulating IGF-1 and glucose homeostasis or are an indirect consequence of DNA damage that accumulates in these mutant mice.

\section{SIRT7 promotes rRNA transcription}

SIRT7 localizes to the nucleolus of human cells (Michishita et al. 2005; Ford et al. 2006). Interestingly, SIRT7 expression correlates with growth (Ford et al. 2006) - it is abundant in tissues with high proliferation, such as liver, spleen, and testes. By contrast, SIRT7 expression is absent or low in nonproliferating tissues, like heart, brain, and muscle.

Recent work has shown that SIRT7 may regulate cellular growth and metabolism (Ford et al. 2006). In the nucleolus, SIRT7 associates with rDNA and interacts with RNA polymerase I (Pol I). Overexpressing SIRT7 increases rRNA transcription and RNA inhibition of SIRT7 decreases transcription, showing that this sirtuin activates Pol I transcription (Ford et al. 2006).

An NAD-dependent deacetylase activity has not been observed for SIRT7 (North et al. 2003), but the amino acid residues that bind NAD in the conserved sirtuin core domain are required for SIRT7 activity (Ford et al. 2006), suggesting a role for NAD-dependent regulation. SIRT7 thus appears to regulate cell growth and metabolism in response to changing metabolic conditions by driving ribosome biogenesis in dividing cells. It is interesting that both SIRT7, as an activator of rRNA transcription, and SIRT1, as an inhibitor of p53 and FOXO, have features that are progrowth and prosurvival for cells.

\section{Cytoplasmic sirtuins}

To date, only SIRT2 is reported to be localized mainly in the cytoplasm (North et al. 2003; Michishita et al. 2005), while a fraction of SIRT2 is nuclear (North et al. 2003). Interestingly, SIRT1 is also reported to be a cytoplasmic protein in pancreatic $\alpha$-cells (Imai et al. 2000). These findings lead us to speculate that mammalian sirtuins 
may shuttle between the nucleus and cytoplasm, depending on cell type or environmental stimuli.

\section{SIRT2}

Mammalian SIRT2 is a predominantly cytoplasmic protein (Dryden et al. 2003; North et al. 2003; Michishita et al. 2005), colocalizes with tubulin, and can deacetylate a number of substrates in vitro, including $\alpha$-tubulin (North et al. 2003) and histones, although the physiological consequences of $\alpha$-tubulin deacetylation by SIRT2 are not yet clear. The yeast ortholog of SIRT2, Hst2, can function in parallel to SIR2 in certain strains with respect to lifespan extension and rDNA silencing (Lamming et al. 2005). Therefore, it will be interesting to determine the lifespan of SIRT2-overexpressing mice, or to determine whether CR is partly mediated by SIRT2 using SIRT2 KO animals.

Cell culture studies demonstrate SIRT2 may be important in regulating mammalian cell cycle. SIRT2 protein levels increase during mitotic phase of the cell cycle and its overexpression delays mitosis (Dryden et al. 2003). Consistent with the idea that SIRT2 may restrain the cell cycle, expression of this sirtuin is down-regulated in human gliomas, compared with normal brain samples (Hiratsuka et al. 2003). SIRT2 colocalizes with chromatin during the $\mathrm{G}_{2} / \mathrm{M}$ transition, a period in which the nuclear membrane has broken down (Vaquero et al. 2006). Both SIRT2 and Hst2 show a preference for deacetylating histone $\mathrm{H} 4$ at Lys16 in vitro, and SIRT2 KO mouse embryonic fibroblasts (MEFs) display hyperacetylated H4K16 during mitosis. Since SIRT1 also deacetylates H4K16, SIRT2 and SIRT1 may function redundantly, at least during the $\mathrm{M}$ phase of the cell cycle. SIRT2 may also regulate other phases of the cell cycle, since $G_{1}$ is extended and $S$ is shortened in SIRT2 ${ }^{-/-}$ MEFs.

\section{Mitochondrial sirtuins-key regulators of metabolism}

Mitochondria are dynamic organelles that regulate nutrient utilization to provide the cell with energy even during dramatic changes in diet and development. Mitochondria also play a central role in mediating apoptosis in response to DNA damage or oxidative stress. These organelles are the primary site of reactive oxygen species (ROS) generation within the cell, and increased oxidative damage is proposed to be one cause of mammalian aging (Harmon 1956; Wallace 2005).

The mitochondrial localization of SIRT3-5 is especially intriguing because mitochondrial dysfunction is associated with mammalian aging and many diseases, including diabetes, neurodegenerative diseases, and cancer (Wallace 2005). Do mitochondrial sirtuins regulate metabolism, the oxidative stress response, and ultimately, mammalian aging? It is important to note that although SIRT1 is not itself physically associated with mitochondria, as described above, it also impacts mitochondrial functions. Lifespan analysis of animals with varying SIRT3-5 level has not been performed; however, there is growing evidence linking mitochondrial sirtuins with regulating energy usage and even human lifespan.

\section{SIRT3}

SIRT3 was the first sirtuin shown to be localized to the mitochondria of mammalian cells (Onyango et al. 2002; Schwer et al. 2002; Michishita et al. 2005). SIRT3 is localized to the mitochondrial matrix and cleavage of its signal sequence is necessary for enzymatic activity (Schwer et al. 2002). SIRT3 deacetylates multiple substrates in vitro including histone peptides (Onyango et al. 2002; Schwer et al. 2002) and tubulin (North et al. 2003).

The biological functions of SIRT3 are beginning to emerge. SIRT3 is expressed in brown adipose tissue and induced by cold exposure (Shi et al. 2005). Moreover, the deacetylase activity of SIRT3 is reported to be required for the induction of uncoupling protein 1 (UCP-1). SIRT3 also appears to regulate mitochondrial functions, as its overexpression increases respiration, while decreasing reactive oxygen species production (Shi et al. 2005).

Two recent studies demonstrate that SIRT3 may regulate the activity of acetyl-CoA synthetase (AceCS) (Hallows et al. 2006; Schwer et al. 2006), representing a striking, conserved activity with the bacterial sirtuin, cobB (Tsang and Escalante-Semerena 1998; Starai et al. 2002). AceCS uses acetate, CoA, and ATP to form acetyl-CoA, which is an intermediate in the TCA cycle, and is also required for cholesterol and fatty acid synthesis. Acetylation of mitochondrial AceCS (AceCS2) inactivates the enzyme, whereas deacetylation by SIRT3 activates it. Interestingly, SIRT1 can deacetylate and activate the cytosolic form of AceCS (AceCS1). These data suggest that SIRT3 may play a role in regulating the entry of carbons from acetate into central metabolism. It will be important to assess the in vivo relevance of these findings using SIRT3 ${ }^{-/-}$mice. In sum, SIRT3 may be especially important under conditions of energy limitation-i.e., during fasting or CR to ensure full incorporation of dietary or ketone-derived acetate into metabolism.

In human population studies, polymorphisms within the SIRT3 gene have been linked to longevity. The G477T transversion, while not affecting the amino acid sequence, associates with survivalship of elderly males (Rose et al. 2003) and may signify a haplotype promoting longevity. The same group found that a variable number of tandem repeats (VNTR) enhancer within SIRT3 also associates with lifespans $>90$ yr (Bellizzi et al. 2005). These findings will need to be validated in larger samples, but suggest that the expression of SIRT3 may promote longevity in humans and raise the importance of performing lifespan experiments in mice that overexpress or lack SIRT3.

\section{SIRT4}

SIRT4 is another mitochondrial protein (Michishita et al. 2005) that regulates energy usage. SIRT4 lacks detectable deacetylase activity (North et al. 2003), but demon- 


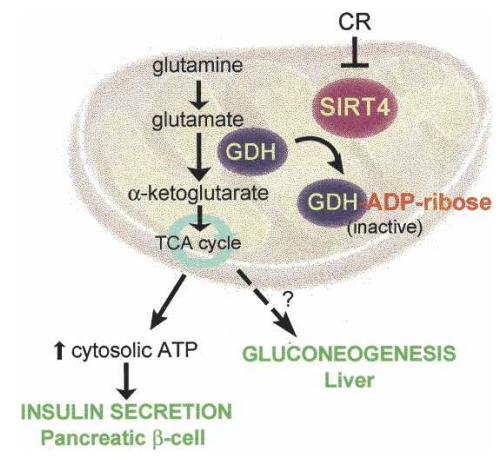

Figure 3. Model of SIRT4 function in pancreatic $\beta$-cells and liver. SIRT4 ADP-ribosylates and inhibits GDH. In pancreatic $\beta$-cells, SIRT4 thus regulates AASIS. In liver, SIRT4 may also regulate the metabolism of amino acids to glucose.

strates ADP-ribosyltransferase activity. SIRT4 plays an important role in regulating amino acid-stimulated insulin secretion (AASIS) in pancreatic $\beta$-cells by ADPribosylating and inhibiting glutamate dehydrogenase (GDH) (Fig. 3; Haigis et al. 2006). GDH converts glutamate into $\alpha$-ketoglutarate, a TCA cycle intermediate. GDH-activating mutations cause hyperinsulinism in humans showing that this enzyme regulates insulin secretion by gating the flow of amino acids into central metabolism in $\beta$-cells (Stanley et al. 1998). SIRT4 KO mice have no gross abnormalities, but display higher GDH activity and higher levels of circulating insulin. SIRT4 KO mice have elevated AASIS, and strikingly, unlike wild type, they secrete insulin in response to glutamine.

SIRT4 may also connect insulin secretion with CR. Islets isolated from CR mice demonstrate increased AASIS similar to islets from ad libitum fed SIRT4 KO mice (Haigis et al. 2006). Down-regulation of SIRT4 during CR appears to mediate this effect because GDH from islets of $\mathrm{CR}$ mice is less ADP-ribosylated and more active than GDH from control islets. A similar change in GDH is found in the liver of CR mice. We suggest that SIRT4 coordinates a physiological response in liver and $\beta$-cells during energy limitation (Fig. 3). In the liver, the flow of carbon from amino acids into gluconeogenesis would be increased, and in $\beta$-cells the ability amino acids to trigger insulin secretion would be elevated. Since overall insulin secretion is clearly lower during CR because of reduced blood glucose, it seems likely that the spectrum of insulin secretagogues is thus shifted from carbohydrates to amino acids.

We note that the apparent down-regulation of SIRT4 during $\mathrm{CR}$ in $\beta$-cells and liver goes against the expectation that sirtuin activity should increase during this dietary regimen. However, it is consistent with the observed reduction in the NAD/NADH ratio in liver of CR mice (Hagopian et al. 2003b) and the observed increase in gluconeogenesis in this organ (Hagopian et al. 2003a). We suggest that a shift from carbohydrates to fat as preferred energy source during CR may help drive down the NAD/ $\mathrm{NADH}$ ratio in these tissues and thus moderate these metabolic changes.

\section{SIRT5}

SIRT5 remains the least characterized sirtuin to date. SIRT5 is described as a mitochondrial protein (Michishita et al. 2005) and has weak deacetylase activity (North et al. 2003), but does not appear to possess an ADP-ribosyltransferase activity (Haigis et al. 2006). Possible physiological substrates of SIRT5 and its role in mammalian cells are not yet known.

\section{Summary and perspective}

Sirtuins have emerged as key antiaging genes in model organisms. The NAD-dependence of these proteins links them unavoidably to the metabolic activity of cells. In several organisms, sirtuins have been shown to be regulated by and to mediate the effects of the dietary regimen CR. Moreover, mammalian sirtuins have been implicated in stress resistance and numerous metabolic pathways, including adipogenesis, gluconeogenesis, and insulin and glucose homeostasis.

While it may be years before we know whether sirtuins regulate mammalian lifespan, current data suggests that these proteins are regulated by diet and in turn, regulate multiple facets of physiology, making them interesting therapeutic targets for metabolic and neurodegenerative diseases (Fig. 4). Several important questions need to be resolved to improve our understanding of sirtuin biology and their therapeutic potential. First, what other functions can be ascribed to the SIRT1-7 proteins? We predict that like SIRT1, SIRT2-7 will have multiple targets and may affect many biologies. Second, how is sirtuin function regulated? Two known mechanisms of sirtuin regulation are (1) its protein induction during CR

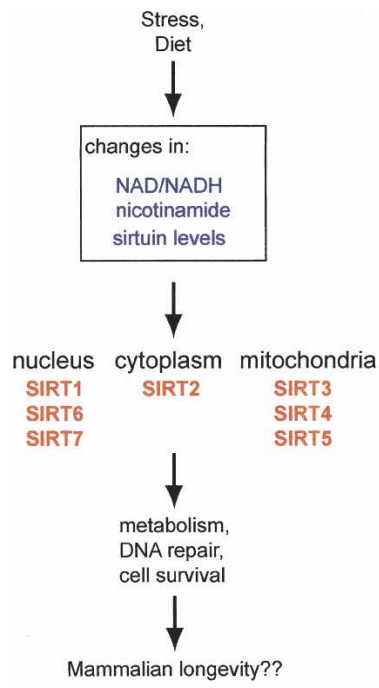

Figure 4. Model of Sirtuin regulation and function. Changes in environmental stress and diet may regulate sirtuin activity by altering the NAD/NADH ratio, nicotinamide levels, or sirtuin levels. Mammalian sirtuins (SIRT1-7) have individual targets and roles in the nucleus, cytoplasm, or mitochondria, and may uniquely impact metabolism, DNA repair, or cell survival. 
or fasting (Cohen et al. 2004; Nisoli et al. 2005; Rodgers et al. 2005) and (2) regulation by metabolites NAD, $\mathrm{NADH}$, and nicotinamide (Anderson et al. 2003a,b; Lin et al. 2004). Third, are all seven sirtuins regulated in the same direction by $\mathrm{CR}$ in any given tissue? If the NAD/ $\mathrm{NADH}$ ratio (or nicotinamide concentration) is a primary determinant of regulation, it is possible that all seven sirtuins will be regulated similarly by $\mathrm{CR}$ in a given cell, since the NAD/NADH ratio has the potential to equilibrate throughout cellular compartments by shuttle systems. Another likely possibility is that the concentration of NAD and its metabolites are regulated compartmentally. For example, enzymes involved in NAD biosynthesis are found in the nucleus, peroxisome, Golgi apparatus, mitochondria, and cytoplasm (Yang et al. 2006). Fourth, does the NAD/NADH ratio change in different directions depending on the tissue in response to CR? This possibility seems likely, since CR induces distinct metabolic changes in different tissues (e.g., activation of gluconeogenesis in liver and fat loss in white adipose tissue). Moreover, fasting decreases SIRT1 activity in the pancreas (Bordone et al. 2006), while increasing its activity in the liver (Rodgers et al. 2005). This last question is important, because if sirtuins are activated by $\mathrm{CR}$ in some tissues but repressed in others, genetically altered mice (i.e., SIRT knockout or overexpressed) or pharmacological interventions to activate or repress a sirtuin systemically (see below) may mimic CR in only a segmental fashion.

In the next few years the answers to these and other questions will auger how well pharmacological agents that target sirtuins will serve as CR mimetics. This path of drug intervention is especially compelling, because CR mitigates many major diseases in rodent models. We can hope that new classes of drugs are on the horizon to deliver broad benefits for these diseases.

\section{References}

Accili, D. and Arden, K.C. 2004. FoxOs at the crossroads of cellular metabolism, differentiation, and transformation. Cell 117: 421-426.

Anderson, R.M., Bitterman, K.J., Wood, J.G., Medvedik, O., and Sinclair, D.A. 2003a. Nicotinamide and PNC1 govern lifespan extension by calorie restriction in Saccharomyces cerevisiae. Nature 423: 181-185.

Anderson, R.M., Latorre-Esteves, M., Neves, A.R., Lavu, S., Medvedik, O., Taylor, C., Howitz, K.T., Santos, H., and Sinclair, D.A. 2003b. Yeast life-span extension by calorie restriction is independent of NAD fluctuation. Science 302: 2124-2126.

Araki, T., Sasaki, Y., and Milbrandt, J. 2004. Increased nuclear NAD biosynthesis and SIRT1 activation prevent axonal degeneration. Science 305: 1010-1013.

Austad, S.N. 1989. Life extension by dietary restriction in the bowl and doily spider, Frontinella pyramitela. Exp. Gerontol. 24: 83-92.

Barzilai, N., Banerjee, S., Hawkins, M., Chen, W., and Rossetti, L. 1998. Caloric restriction reverses hepatic insulin resistance in aging rats by decreasing visceral fat. J. Clin. Invest. 101: 1353-1361.

Bellizzi, D., Rose, G., Cavalcante, P., Covello, G., Dato, S., De
Rango, F., Greco, V., Maggiolini, M., Feraco, E., Mari, V., et al. 2005. A novel VNTR enhancer within the SIRT3 gene, a human homologue of SIR2, is associated with survival at oldest ages. Genomics 85: 258-263.

Berdichevsky, A., Viswanathan, M., Horvitz, H.R., and Guarente, L. 2006. C. elegans SIR-2.1 interacts with 14-3-3 proteins to activate DAF-16 and extend life span. Cell 125: 1165-1177.

Blander, G. and Guarente, L. 2004. The Sir2 family of protein deacetylases. Annu. Rev. Biochem. 73: 417-435.

Bordone, L., Motta, M.C., Picard, F., Robinson, A., Jhala, U.S., Apfeld, J., McDonagh, T., Lemieux, M., McBurney, M., Szilvasi, A., et al. 2006. Sirt1 regulates insulin secretion by repressing UCP2 in pancreatic $\beta$ cells. PLoS Biol. 4: 31 .

Brunet, A., Sweeney, L.B., Sturgill, J.F., Chua, K.F., Greer, P.L., Lin, Y., Tran, H., Ross, S.E., Mostoslavsky, R., Cohen, H.Y., et al. 2004. Stress-dependent regulation of FOXO transcription factors by the SIRT1 deacetylase. Science 303: 20112015.

Chen, D., Steele, A.D., Lindquist, S., and Guarente, L. 2005. Increase in activity during calorie restriction requires Sirt1. Science 310: 1641.

Chen, J., Zhou, Y., Mueller-Steiner, S., Chen, L.F., Kwon, H., Yi, S., Mucke, L., and Gan, L. 2005. SIRT1 protects against microglia-dependent amyloid- $\beta$ toxicity through inhibiting NFкB signaling. J. Biol. Chem. 280: 40364-40374.

Cheng, H.L., Mostoslavsky, R., Saito, S., Manis, J.P., Gu, Y., Patel, P., Bronson, R., Appella, E., Alt, F.W., and Chua, K.F. 2003. Developmental defects and p53 hyperacetylation in Sir2 homolog (SIRT1)-deficient mice. Proc. Natl. Acad. Sci. 100: 10794-10799.

Chua, K.F., Mostoslavsky, R., Lombard, D.B., Pang, W.W., Saito, S., Franco, S., Kaushal, D., Cheng, H.L., Fischer, M.R., Stokes, N., et al. 2005. Mammalian SIRT1 limits replicative life span in response to chronic genotoxic stress. Cell Metab. 2: $67-76$.

Cohen, H.Y., Miller, C., Bitterman, K.J., Wall, N.R., Hekking, B., Kessler, B., Howitz, K.T., Gorospe, M., de Cabo, R., and Sinclair, D.A. 2004. Calorie restriction promotes mammalian cell survival by inducing the SIRT1 deacetylase. Science 305: 390-392.

Comfort, A. 1963. Effect of delayed and resumed growth on the longevity of a fish (Lebistes reticulatus, Peters) in captivity. Gerontologia 49: 150-155.

Dhahbi, J.M., Mote, P.L., Wingo, J., Rowley, B.C., Cao, S.X., Walford, R.L., and Spindler, S.R. 2001. Caloric restriction alters the feeding response of key metabolic enzyme genes. Mech. Ageing Dev. 122: 1033-1048.

Dryden, S.C., Nahhas, F.A., Nowak, J.E., Goustin, A.S., and Tainsky, M.A. 2003. Role for human SIRT2 NAD-dependent deacetylase activity in control of mitotic exit in the cell cycle. Mol. Cell. Biol. 23: 3173-3185.

Duan, W. and Mattson, M.P. 1999. Dietary restriction and 2-deoxyglucose administration improve behavioral outcome and reduce degeneration of dopaminergic neurons in models of Parkinson's disease. J. Neurosci. Res. 57: 195-206.

Fabrizio, P., Gattazzo, C., Battistella, L., Wei, M., Cheng, C., McGrew, K., and Longo, V.D. 2005. Sir2 blocks extreme lifespan extension. Cell 123: 655-667.

Ford, E., Voit, R., Liszt, G., Magin, C., Grummt, I., and Guarente, L. 2006. Mammalian Sir2 homolog SIRT7 is an activator of RNA polymerase I transcription. Genes \& Dev. 20: $1075-1080$.

Frescas, D., Valenti, L., and Accili, D. 2005. Nuclear trapping of the forkhead transcription factor FoxO1 via Sirt-dependent deacetylation promotes expression of glucogenetic genes. $J$. 
Biol. Chem. 280: 20589-20595.

Frye, R.A. 1999. Characterization of five human cDNAs with homology to the yeast SIR2 gene: Sir2-like proteins (sirtuins) metabolize NAD and may have protein ADP-ribosyltransferase activity. Biochem. Biophys. Res. Commun. 260: 273 279

Frye, R.A. 2000. Phylogenetic classification of prokaryotic and eukaryotic Sir2-like proteins. Biochem. Biophys. Res. Commun. 273: 793-798.

Fulco, M., Schiltz, R.L., Iezzi, S., King, M.T., Zhao, P., Kashiwaya, Y., Hoffman, E., Veech, R.L., and Sartorelli, V. 2003. Sir2 regulates skeletal muscle differentiation as a potential sensor of the redox state. Mol. Cell 12: 51-62.

Grubisha, O., Smith, B.C., and Denu, J.M. 2005. Small molecule regulation of Sir2 protein deacetylases. FEBS J. 272: 46074616.

Grubisha, O., Rafty, L.A., Takanishi, C.L., Xu, X., Tong, L., Perraud, A.L., Scharenberg, A.M., and Denu, J.M. 2006. Metabolite of SIR2 reaction modulates TRPM2 ion channel. J. Biol. Chem. 281: 14057-14065.

Hagopian, K., Ramsey, J.J., and Weindruch, R. 2003a. Caloric restriction increases gluconeogenic and transaminase enzyme activities in mouse liver. Exp. Gerontol. 38: 267-278.

Hagopian, K., Ramsey, J.J., and Weindruch, R. 2003b. Influence of age and caloric restriction on liver glycolytic enzyme activities and metabolite concentrations in mice. Exp. Gerontol. 38: 253-266.

Haigis, M.C., Mostoslavsky, R., Haigis, K.M., Fahie, K., Christodoulou, D.C., Murphy, A.J., Valenzuela, D.M., Yancopoulos, G.D., Karow, M., Blander, G., et al. 2006. SIRT4 inhibits glutamate dehydrogenase and opposes the effects of calorie restriction in pancreatic $\beta$ cells. Cell 126: 941-954.

Hallows, W.C., Lee, S., and Denu, J.M. 2006. Sirtuins deacetylate and activate mammalian acetyl-CoA synthetases. Proc. Natl. Acad. Sci. 103: 10230-10235.

Harmon, D. 1956. Aging: A theory based on free radical and radiation chemistry. J. Gerontol. 11: 298.

Hiratsuka, M., Inoue, T., Toda, T., Kimura, N., Shirayoshi, Y., Kamitani, H., Watanabe, T., Ohama, E., Tahimic, C.G., Kurimasa, A., et al. 2003. Proteomics-based identification of differentially expressed genes in human gliomas: Down-regulation of SIRT2 gene. Biochem. Biophys. Res. Commun. 309: 558-566.

Howitz, K.T., Bitterman, K.J., Cohen, H.Y., Lamming, D.W., Lavu, S., Wood, J.G., Zipkin, R.E., Chung, P., Kisielewski, A., Zhang, L.L., et al. 2003. Small molecule activators of sirtuins extend Saccharomyces cerevisiae lifespan. Nature 425: 191196.

Imai, S., Johnson, F.B., Marciniak, R.A., McVey, M., Park, P.U., and Guarente, L. 2000. Sir2: An NAD-dependent histone deacetylase that connects chromatin silencing, metabolism, and aging. Cold Spring Harb. Symp. Quant. Biol. 65: 297302.

Kaeberlein, M., McVey, M., and Guarente, L. 1999. The SIR2/ $3 / 4$ complex and SIR2 alone promote longevity in Saccharomyces cerevisiae by two different mechanisms. Genes \& Dev. 13: 2570-2580.

Kaeberlein, M., Kirkland, K.T., Fields, S., and Kennedy, B.K. 2004. Sir2-independent life span extension by calorie restriction in yeast. PLOS Biol. 2: E296.

Kaeberlein, M., Hu, D., Kerr, E.O., Tsuchiya, M., Westman, E.A., Dang, N., Fields, S., and Kennedy, B.K. 2005. Increased life span due to calorie restriction in respiratory-deficient yeast. PLoS Genet. 1: e69.

Kitamura, Y.I., Kitamura, T., Kruse, J.P., Raum, J.C., Stein, R., $\mathrm{Gu}, \mathrm{W}$., and Accili, D. 2005. FoxO1 protects against pancre- atic $\beta$ cell failure through NeuroD and MafA induction. Cell Metab. 2: 153-163.

Lamming, D.W., Latorre-Esteves, M., Medvedik, O., Wong, S.N., Tsang, F.A., Wang, C., Lin, S.J., and Sinclair, D.A. 2005. HST2 mediates SIR2-independent life-span extension by calorie restriction. Science 309: 1861-1864.

Landry, J., Sutton, A., Tafrov, S.T., Heller, R.C., Stebbins, J., Pillus, L., and Sternglanz, R. 2000. The silencing protein SIR2 and its homologs are NAD-dependent protein deacetylases. Proc. Natl. Acad. Sci. 97: 5807-5811.

Lemieux, M.E., Yang, X., Jardine, K., He, X., Jacobsen, K.X., Staines, W.A., Harper, M.E., and McBurney, M.W. 2005. The Sirt 1 deacetylase modulates the insulin-like growth factor signaling pathway in mammals. Mech. Ageing Dev. 126: 1097-1105.

Lin, S.J., Defossez, P.A., and Guarente, L. 2000. Requirement of NAD and SIR2 for life-span extension by calorie restriction in Saccharomyces cerevisiae. Science 289: 2126-2128.

Lin, S.J., Kaeberlein, M., Andalis, A.A., Sturtz, L.A., Defossez, P.A., Culotta, V.C., Fink, G.R., and Guarente, L. 2002. Calorie restriction extends Saccharomyces cerevisiae lifespan by increasing respiration. Nature 418: 344-348.

Lin, S.J., Ford, E., Haigis, M., Liszt, G., and Guarente, L. 2004. Calorie restriction extends yeast life span by lowering the level of NADH. Genes \& Dev. 18: 12-16.

Liszt, G., Ford, E., Kurtev, M., and Guarente, L. 2005. Mouse Sir2 homolog SIRT6 is a nuclear ADP-ribosyltransferase. J. Biol. Chem. 280: 21313-21320.

Loeb, J. and Northrop, J.H. 1917. On the influence of food and temperature upon the duration of life. J. Biol. Chem. 32: 103-121.

Luo, J., Nikolaev, A.Y., Imai, S., Chen, D., Su, F., Shiloh, A., Guarente, L., and Gu, W. 2001. Negative control of p53 by Sir2 $\alpha$ promotes cell survival under stress. Cell 107: 137-148.

McBurney, M.W., Yang, X., Jardine, K., Hixon, M., Boekelheide, K., Webb, J.R., Lansdorp, P.M., and Lemieux, M. 2003. The mammalian SIR $2 \alpha$ protein has a role in embryogenesis and gametogenesis. Mol. Cell. Biol. 23: 38-54.

McCay, C.M., Crowell, M.F., and Maynard, L.A. 1935. The effect of retarded growth upon the length of life span and upon the ultimate body size. J. Nutr. 10: 63-79.

Michishita, E., Park, J.Y., Burneskis, J.M., Barrett, J.C., and Horikawa, I. 2005. Evolutionarily conserved and nonconserved cellular localizations and functions of human SIRT proteins. Mol. Biol. Cell 16: 4623-4635.

Morley, J.F., Brignull, H.R., Weyers, J.J., and Morimoto, R.I. 2002. The threshold for polyglutamine-expansion protein aggregation and cellular toxicity is dynamic and influenced by aging in Caenorhabditis elegans. Proc. Natl. Acad. Sci. 99: 10417-10422.

Mostoslavsky, R., Chua, K.F., Lombard, D.B., Pang, W.W., Fischer, M.R., Gellon, L., Liu, P., Mostoslavsky, G., Franco, S., Murphy, M.M., et al. 2006. Genomic instability and aging-like phenotype in the absence of mammalian SIRT6. Cell 124: 315-329.

Motta, M.C., Divecha, N., Lemieux, M., Kamel, C., Chen, D., Gu, W., Bultsma, Y., McBurney, M., and Guarente, L. 2004. Mammalian SIRT1 represses forkhead transcription factors. Cell 116: 551-563.

Moynihan, K.A., Grimm, A.A., Plueger, M.M., Bernal-Mizrachi, E., Ford, E., Cras-Meneur, C., Permutt, M.A., and Imai, S. 2005. Increased dosage of mammalian Sir2 in pancreatic $\beta$ cells enhances glucose-stimulated insulin secretion in mice. Cell Metab. 2: 105-117.

Nemoto, S., Fergusson, M.M., and Finkel, T. 2005. SIRT1 functionally interacts with the metabolic regulator and transcrip- 
tional coactivator PGC-1 $\alpha$. J. Biol. Chem. 280: 16456-16460.

Nisoli, E., Tonello, C., Cardile, A., Cozzi, V., Bracale, R., Tedesco, L., Falcone, S., Valerio, A., Cantoni, O., Clementi, E., et al. 2005. Calorie restriction promotes mitochondrial biogenesis by inducing the expression of eNOS. Science 310: 314-317.

North, B.J., Marshall, B.L., Borra, M.T., Denu, J.M., and Verdin, E. 2003. The human Sir2 ortholog, SIRT2, is an NAD ${ }^{+}$-dependent tubulin deacetylase. Mol. Cell 11: 437-444.

Onyango, P., Celic, I., McCaffery, J.M., Boeke, J.D., and Feinberg, A.P. 2002. SIRT3, a human SIR2 homologue, is an NAD-dependent deacetylase localized to mitochondria. Proc. Nat1. Acad. Sci. 99: 13653-13658.

Parker, J.A., Arango, M., Abderrahmane, S., Lambert, E., Tourette, C., Catoire, H., and Neri, C. 2005. Resveratrol rescues mutant polyglutamine cytotoxicity in nematode and mammalian neurons. Nat. Genet. 37: 349-350.

Patel, N.V., Gordon, M.N., Connor, K.E., Good, R.A., Engelman, R.W., Mason, J., Morgan, D.G., Morgan, T.E., and Finch, C.E. 2005. Caloric restriction attenuates A $\beta$-deposition in Alzheimer transgenic models. Neurobiol. Aging 26: 995-1000.

Picard, F., Kurtev, M., Chung, N., Topark-Ngarm, A., Senawong, T., Machado De Oliveira, R., Leid, M., McBurney, M.W., and Guarente, L. 2004. Sirt1 promotes fat mobilization in white adipocytes by repressing PPAR- $\gamma$. Nature 429: 771-776.

Rodgers, J.T., Lerin, C., Haas, W., Gygi, S.P., Spiegelman, B.M., and Puigserver, P. 2005. Nutrient control of glucose homeostasis through a complex of PGC-1 $\alpha$ and SIRT1. Nature 434: $113-118$.

Rogina, B. and Helfand, S.L. 2004. Sir2 mediates longevity in the fly through a pathway related to calorie restriction. Proc. Nat1. Acad. Sci. 101: 15998-16003.

Rose, G., Dato, S., Altomare, K., Bellizzi, D., Garasto, S., Greco, V., Passarino, G., Feraco, E., Mari, V., Barbi, C., et al. 2003. Variability of the SIRT3 gene, human silent information regulator Sir2 homologue, and survivorship in the elderly. Exp. Gerontol. 38: 1065-1070.

Sauve, A.A., Celic, I., Avalos, J., Deng, H., Boeke, J.D., and Schramm, V.L. 2001. Chemistry of gene silencing: The mechanism of $\mathrm{NAD}^{+}$-dependent deacetylation reactions. Biochemistry 40: 15456-15463.

Schwer, B., North, B.J., Frye, R.A., Ott, M., and Verdin, E. 2002. The human silent information regulator (Sir)2 homologue hSIRT3 is a mitochondrial nicotinamide adenine dinucleotide-dependent deacetylase. J. Cell Biol. 158: 647-657.

Schwer, B., Bunkenborg, J., Verdin, R.O., Andersen, J.S., and Verdin, E. 2006. Reversible lysine acetylation controls the activity of the mitochondrial enzyme acetyl-CoA synthetase 2. Proc. Nat1. Acad. Sci. 103: 10224-10229.

Shi, T., Wang, F., Stieren, E., and Tong, Q. 2005. SIRT3, a mitochondrial sirtuin deacetylase, regulates mitochondrial function and thermogenesis in brown adipocytes. J. Biol. Chem. 280: 13560-13567.

Sinclair, D.A. and Guarente, L. 1997. Extrachromosomal rDNA circles-A cause of aging in yeast. Cell 91: 1033-1042.

Smith, J.S., Brachmann, C.B., Celic, I., Kenna, M.A., Muhammad, S., Starai, V.J., Avalos, J.L., Escalante-Semerena, J.C., Grubmeyer, C., Wolberger, C., et al. 2000. A phylogenetically conserved $\mathrm{NAD}^{+}$-dependent protein deacetylase activity in the Sir2 protein family. Proc. Natl. Acad. Sci. 97: 6658-6663.

Stanley, C.A., Lieu, Y.K., Hsu, B.Y., Burlina, A.B., Greenberg, C.R., Hopwood, N.J., Perlman, K., Rich, B.H., Zammarchi, E., and Poncz, M. 1998. Hyperinsulinism and hyperammo- nemia in infants with regulatory mutations of the glutamate dehydrogenase gene. N. Engl. J. Med. 338: 1352-1357.

Starai, V.J., Celic, I., Cole, R.N., Boeke, J.D., and EscalanteSemerena, J.C. 2002. Sir2-dependent activation of acetylCoA synthetase by deacetylation of active lysine. Science 298: 2390-2392.

Tanner, K.G., Landry, J., Sternglanz, R., and Denu, J.M. 2000. Silent information regulator 2 family of NAD-dependent histone/protein deacetylases generates a unique product, 1-Oacetyl-ADP-ribose. Proc. Natl. Acad. Sci. 97: 14178-14182.

Tanny, J.C. and Moazed, D. 2001. Coupling of histone deacetylation to NAD breakdown by the yeast silencing protein Sir2: Evidence for acetyl transfer from substrate to an NAD breakdown product. Proc. Natl. Acad. Sci. 98: 415-420.

Tanny, J.C., Dowd, G.J., Huang, J., Hilz, H., and Moazed, D. 1999. An enzymatic activity in the yeast Sir2 protein that is essential for gene silencing. Cell 99: 735-745.

Tissenbaum, H.A. and Guarente, L. 2001. Increased dosage of a sir-2 gene extends lifespan in Caenorhabditis elegans. $\mathrm{Na}$ ture 410: 227-230.

Tsang, A.W. and Escalante-Semerena, J.C. 1998. CobB, a new member of the SIR 2 family of eucaryotic regulatory proteins, is required to compensate for the lack of nicotinate mononucleotide:5,6-dimethylbenzimidazole phosphoribosyltransferase activity in cobT mutants during cobalamin biosynthesis in Salmonella typhimurium LT2. J. Biol. Chem. 273: 31788-31794.

Vaquero, A., Scher, M.B., Lee, D.H., Sutton, A., Cheng, H.L., Alt, F.W., Serrano, L., Sternglanz, R., and Reinberg, D. 2006. SirT2 is a histone deacetylase with preference for histone $\mathrm{H} 4$ Lys 16 during mitosis. Genes \& Dev. 20: 1256-1261.

Vaziri, H., Dessain, S.K., Ng Eaton, E., Imai, S.I., Frye, R.A., Pandita, T.K., Guarente, L., and Weinberg, R.A. 2001. hSIR2(SIRT1) functions as an NAD-dependent p53 deacetylase. Cell 107: 149-159.

Wallace, D.C. 2005. A mitochondrial paradigm of metabolic and degenerative diseases, aging, and cancer: A dawn for evolutionary medicine. Annu. Rev. Genet. 39: 359-407.

Wang, Y. and Tissenbaum, H.A. 2006. Overlapping and distinct functions for a Caenorhabditis elegans SIR2 and DAF-16/ FOXO. Mech. Ageing Dev. 127: 48-56.

Wang, J., Zhai, Q., Chen, Y., Lin, E., Gu, W., McBurney, M.W., and $\mathrm{He}, \mathrm{Z}$. 2005. A local mechanism mediates NAD-dependent protection of axon degeneration. J. Cell Biol. 170: 349355.

Yang, H., Lavu, S., and Sinclair, D. 2006. Nampt/PBEF/Visfatin: A regulator of mammalian health and longevity? Exp. Gerontol. 41: 718-726.

Yeung, F., Hoberg, J.E., Ramsey, C.S., Keller, M.D., Jones, D.R., Frye, R.A., and Mayo, M.W. 2004. Modulation of NF-кBdependent transcription and cell survival by the SIRT1 deacetylase. EMBO T. 23: 2369-2380.

Zhu, H., Guo, Q., and Mattson, M.P. 1999. Dietary restriction protects hippocampal neurons against the death-promoting action of a presenilin-1 mutation. Brain Res. 842: 224-229. 


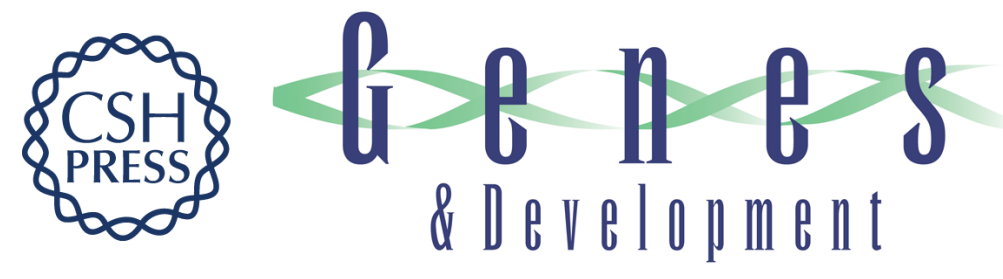

\section{Mammalian sirtuins--emerging roles in physiology, aging, and calorie restriction}

Marcia C. Haigis and Leonard P. Guarente

Genes Dev. 2006, 20:

Access the most recent version at doi:10.1101/gad.1467506

$\begin{array}{ll}\text { References } & \text { This article cites } 87 \text { articles, } 38 \text { of which can be accessed free at: } \\ \text { http://genesdev.cshlp.org/content/20/21/2913.full.html\#ref-list-1 }\end{array}$

License

Email Alerting Receive free email alerts when new articles cite this article - sign up in the box at the top Service right corner of the article or click here.

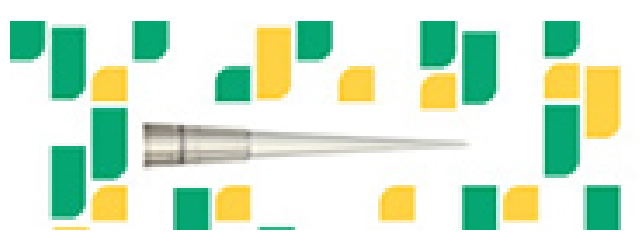

Focused on your science. 